\title{
CÁLCULO EN LA DOSIFICACIÓN DE DULCAMARA KALANCHOE GASTONIS BONNIERI SOBRE PARÁMETROS PRODUCTIVOS EN POLLOS EN MORONA SANTIAGO, ECUADOR.
}

\section{Calculation of dulcamara kalanchoe gastonis bonnieri dosage on broiler production parameters in Morona Santiago, Ecuador.}

\author{
${ }^{1}$ Víctor Huebla Concha (iD), ${ }^{1}$ José-Hernán Negrete* (D), ${ }^{1}$ Santiago-Nathanael Toapanta iD, ${ }^{2}$ Luis-Alfonso \\ Condo iD, ${ }^{3}$ Erenkan Lucero Wamputsrik \\ ${ }^{1}$ Escuela Superior Politécnica de Chimborazo, Facultad de Ciencias Pecuarias, Ingeniería en Zootecnia, \\ Macas, Ecuador. \\ ${ }^{2}$ Universidad Regional Amazónica IKIAM, Facultad Socioambiental, Ingeniería en Biocomercio, Tena, \\ Ecuador. \\ ${ }^{3}$ Investigador Independiente, Macas, Ecuador. \\ *jose.negrete@espoch.edu.ec
}

$\mathrm{R}$ esumen

En la provincia de Morona Santiago, se utilizó 4\%, 8\% y $12 \%$ de extracto de Dulcamara Kalanchoe gastonis bonnieri en el agua de bebida en pollos broilers de la línea COBB 500 durante la etapa de crecimiento y engorde con el objetivo de reducir su morbilidad y mortalidad frente a un tratamiento de control para reducir costos de producción. El diseño de la investigación fue experimental, cuantitativa, analítica. En 100 aves de un día de edad se aplicó un diseño al azar con 4 tratamientos, cada uno de ellos con 5 repeticiones bajo condiciones controladas; para el análisis de información se utilizó el Análisis de Varianza, y el método Tukey. Estadísticamente no se registraron diferencias significativas; pero con el extracto de dulcamara al $4 \%$ se obtuvo un peso final de 2.690,88 g; 2.646,92 g de peso ganado y 1,97 de conversión alimenticia sin presentar mortalidad y morbilidad a excepción del tratamiento de control. Al emplear el extracto de Dulcamara también se registró un beneficio/ costo de 1,39 dólares al disminuir costos de producción y generar mayor rentabilidad. Se concluye que es recomendable utillizar extracto de Dulcamara al 4\% como una opción en la producción de pollos broilers.

Palabras claves: Kalanchoe gastonis bonnieri, dulcamara, salud animal, pollos de engorde.

\section{A bstract}

In Morona Santiago province, $4 \%, 8 \%$ and $12 \%$ extract of Dulcamara Kalanchoe gastonis bonnieri was used in drinking water in broiler chickens of the COBB 500 line during the growth and fattening stage in order to reduce their morbidity and mortality compared to a control treatment to reduce production costs. The research design was experimental, quantitative, analytical. A random design with 4 treatments was applied to 100 one-day-old birds, each with 5 repetitions under controlled conditions; Analysis of Variance and the Tukey method were used for information analysis. Statistically there were no significant differences; but with the $4 \%$ dulcamara extract a final weight of 2,690.88 $\mathrm{g}$ was obtained; 2,646.92 $\mathrm{g}$ of weight gained and 1.97 of feed conversion without presenting mortality and morbidity except for the control treatment. The use of Dulcamara extract also resulted in a cost/ benefit of US $\$ 1.39$ by reducing production costs and generating higher profitability. It is concluded that it is advisable to use Dulcamara extract at $4 \%$ as an option in broiler production.

Keywords: Kalanchoe gastonis bonnieri, dulcamara, animal health, broilers. 


\section{INTRODUCCIÓN}

La utilización de la Dulcamara para tratamiento o mejoramiento de especies animales ha generado importantes investigaciones internacionales, tal es el caso del estudio denominado "Efficacy of a Kalanchoe gastonis-bonnieri extract to control bacterial biofilms and dental calculus in dogs" en donde se estableció la eficacia de la Dulcamara como agente antimicrobiano contra las bacterias de la cavidad oral canina. Otro estudio importante a nivel nacional es el titulado "Evaluación de la dulcamara (Kalanchoe gastonis bonnieri) en la etapa de crecimiento y engorde en pollos broiler en el barrio Santán del cantón Latacunga" que evaluó la adición de extracto de Dulcamara en agua de bebida para pollos broiler determinando así su eficacia y beneficios.

La demanda en el consumo de pollos en Morona Santiago ha venido creciendo significativamente por lo que la actividad de crianza de pollos broilers o de engorde resulta rentable siempre que se cuente con tiempos menores en la producción, así como carne de calidad y precios competitivos en el mercado.

Para desarrollar esta actividad, usualmente se hace uso de antibióticos, mismos que producen resistencia bacteriana, factor que conlleva un problema para la alimentación de quiénes consumen la carne y a su vez encarece el costo de producción en la crianza de pollos.

La Kalanchoe gastonis-bonnieri se trata de una planta originaria de Madagascar - África, introducida al Ecuador y conocida en la amazonía ecuatoriana como Dulcamara; es utilizada por los nativos como medicina natural. Esta planta contiene alcaloides, triterpenos, glucósidos, flavonoides, esteroides y lípidos, su extracto contiene un grupo de productos químicos llamados bufadienolides, que por su actividad resultan muy apetecidos en la industria de la medicina.

El nombre de Dulcamara se deriva del latín «Dulcamare» que significa agridulce, contiene alcaloides, triterpenos, glucósidos, flavonoides, además de bufadienolides; la investigación denominada "Respuesta del cultivo de dulcamara (Kalanchoe gastonis bonnieri) a la aplicación edáfica complementaria con tres tipos de bioestimulantes" realizada en Cumbayá provincia de Pichincha, se determina que este vegetal tiene un efecto antibacteriano, preventivo antitumores de cáncer (1).

El extracto de Kalanchoe gastonis bonnieri o KGB según lo nominado por Beltrán et al. 2003 (2), fue útil para controlar la microbiota oral de los perros por lo que se determina que podría estar relacionada con la actividad antibacteriana (3). La KGB, es una especie utilizada por las etnias como medicina natural de la familia Crassulaceae, en la medicina latinoamericana es utilizada como anticonceptivo, así como en el tratamiento de infecciones genitales-urinarias y vaginales (4). Entre las muchas propiedades de la KGB están las antioxidantes, inmunomoduladoras y vasodilatadoras, que de acuerdo a lo que menciona la Administración Federal de Drogas y Medicinas de Estados Unidos (FDA), resultan ser prometedoras para controlar algunos tipos de cáncer, tumores, leucemia, lupus, miomas, complicaciones pulmonares y renales, diabetes, bronquitis, úlceras, quemaduras y problemas cutáneos. (5)

La avicultura es una actividad económica importante para la provincia de Morona Santiago, por lo que los productores se ven obligados a mantener estándares altos de productividad. Por esta razón en este estudio se incorporó Dulcamara en las dietas de pollos como un tratamiento antibiótico diferente al tradicional.

Considerando que las propiedades medicinales de la Dulcamara han sido utilizadas para contrarrestar enfermedades presentes en humanos, esta investigación consideró disminuir los índices de morbilidad y mortalidad en pollos de engorde frente a un tratamiento de control para que de esta manera se reduzcan los costos de producción, generando una alternativa de mejoría en la calidad de carne.

\section{MATERIALES Y MÉTODOS}

La presente investigación se desarrolló en el cantón Santiago $\left(2^{\circ} 43^{\prime} 01.2^{\prime \prime} \mathrm{S} 78^{\circ} 19^{\prime} 03.4^{\prime \prime} \mathrm{W}\right)$, provincia de Morona Santiago, Ecuador. El diseño de la investigación fue experimental, analítico y cuantitativo; la dosificación de KGB utilizada fue del $4 \%, 8 \%$ y $12 \%$ frente a un tratamiento de control, el propósito fue establecer calcular el beneficio costo en la producción de pollos Broilers de la línea Cobb 500, así como establecer su nivel de 
productividad sanitaria adecuada.

Para el estudio experimental se trabajó con un galpón con 20 divisiones en donde se distribuyó equitativamente a 100 pollos de un día de edad (línea Cobb 500) de un día de edad. El peso inicial promedio de los pollos era de $43,48 \mathrm{~g}$, a quiénes se les aplicó de forma aleatoria cuatro tratamientos y cinco repeticiones.

Las unidades experimentales contenían 5 pollos tiernos que en total por cada tratamiento sumaban 25 pollos. El fin de esto fue el de comprobar el efecto de la KGB sobre la mortalidad y morbilidad de los pollos. La ecuación utilizaddaa para el cálculo de la muestra fue $n=p q / e 2$, dónde $n:(100)$ es la muestra, $\mathrm{p}(0,5)$ la probabilidad de éxito, $\mathrm{q}$ $(0,5)$ la probabilidad de fracaso y e $(0,05)$ el error admitido.

En cuanto a la dosificación de KGB en el agua de bebida de pollos fue el siguiente: sin extracto de KGB y $100 \%$ de agua (T0); $4 \%$ de KGB y $96 \%$ de agua (T1); $8 \%$ de KGB y $92 \%$ de agua (T2); $12 \%$ KGB y $88 \%$ de agua (T3). En los tratamientos se utilizó un diseño al azar. El análisis de varianza (ADEVA), se utilizó para el análisis de los datos conjuntamente con la prueba de significación estadística de Tukey con una tasa de error del 0,05\%, y análisis de correlación y regresión.

Los parámetros de medición experimental en gramos fueron: Peso inicial, Peso final, Ganancia de peso total, Conversión alimenticia, Peso a la canal; mientras que los parámetros de medición experimental en porcentaje fueron: Rendimiento a la canal, Mortalidad, Morbilidad. El cálculo del beneficio/costo y el económico se realizó en dólares.

\section{RESULTADOS}

El peso inicial promedio tomado utilizando el método gravimétrico de los pollos fue de 43,48 g, siendo homogéneos $(\mathrm{P}>0.21)$.

Un peso inicial de 45,85 g en pollos broilers fue adecuado para suministrar KGB, siendo ligeramente superior al registrado en el presente estudio. Al volver a pesar a los pollos a los 49 días utilizando el método gravimetrico, no se registró diferencias significativas $(\mathrm{P}>0.05)$; sin embargo hay que destacar que con la aplicación de KGB al $4 \%$ el peso máximo de los pollos fue de $2.690,88$ g y el mínimo de $2.571,08 \mathrm{~g}$ que corresponde al tratamiento control.

La ganancia de peso obtenida por diferencias de peso entre el peso a los 49 dias menos el peso inicial no registró diferencias estadísticas significativas ( $\mathrm{P}>0.05)$, sin embargo, al aplicar $4 \%$ de extracto de dulcamara permitió una ganancia de peso de 2.646,92 g y al utilizar dulcamara en pollos registraron $2.691,1 \mathrm{~g}$ de ganancia de peso, al aplicar $1,5 \%$ de extracto de dulcamara y sin dulcamara 2.923,05 g (6).

La relación entre el consumo de alimento y la ganancia de peso más conocido como conversión alimenticia no presentó diferencias significativas $(\mathrm{P}>0.05)$, sin embargo se puede manifestar que al utilizar extracto de dulcamara en dosis de 4\%, alcanzó una eficiencia de alimento de 1,97 seguido del $8 \%$ con el que se obtuvo 1,98 y al aplicar 12 $\%$ de dulcamara se obtuvo 1,99, elevándose a 2,06 los pollos manejados bajo un sistema convencional, de esta manera se puede evidenciar que hubo un efecto positivo de la dulcamara en la eficiencia alimenticia.

\begin{tabular}{|c|c|c|c|c|c|c|}
\hline \multirow{2}{*}{ Variables } & \multicolumn{4}{|c|}{ Tratamientos } & \multirow[t]{2}{*}{ E.E. } & \multirow[t]{2}{*}{ Prob. } \\
\hline & $0 \%$ & $4 \%$ & $8 \%$ & $12 \%$ & & \\
\hline Peso Inicial (g) & 43,44 & 43,96 & 43,16 & 43,36 & 0,20 & 0,21 \\
\hline Peso final (g) & 2571,08 & 2690,88 & 2678,64 & 2652,16 & 0,32 & 0,52 \\
\hline Ganancia de peso (g) & 2527,64 & 2646,92 & 2635,48 & 2608,80 & 0,32 & 0,52 \\
\hline Consumo de alimento (g) & 5200,00 & 5200,00 & 5200,00 & 5200,00 & & \\
\hline Conversión Alimenticia & 2,06 & 1,97 & 1,98 & 1,99 & 0,31 & 0,49 \\
\hline Peso a la canal (g) & 2028,65 & 2092,75 & 2056,72 & 2129,79 & 0,38 & 0,70 \\
\hline Rendimiento a la canal (\%) & 78,85 & 77,73 & 76,74 & 80,30 & 0,20 & 0,19 \\
\hline Mortalidad (\%) & 0,00 & 0,00 & 0,00 & 0,00 & & \\
\hline Morbilidad (\%) & 20,00 & 0,00 & 0,00 & 0,00 & & 0,01 \\
\hline
\end{tabular}

Tabla 1. Comportamientos productivas-sanitarias en pollos broilers por efecto de tres niveles de extracto de dulcamara. 
El peso a la canal de los pollos broilers, no se determinaron diferencias estadísticas, sin embargo, las aves que estuvieron bajo el efecto del 12 $\%$ de dulcamara en el agua de bebida registraron $2.129,79 \mathrm{~g}$ y apenas $2.028,65 \mathrm{~g}$ para el tratamiento control.

El peso a la canal del pollo broiler fue de 2.701 g, (7) al utilizar diferentes relaciones de Lisina: Energía sobre los diferentes parámetros zootécnicos del pollo siendo superiores a los encontrados en el presente trabajo.

El rendimiento a la canal de los pollos broilers no registraron diferencias estadísticas por efecto de diferentes niveles de extracto de dulcamara en agua de bebida, sin embargo, al utilizar $12 \%$ de extracto de dulcamara alcanzaron el 80,30 \% de rendimiento a la canal, seguido del tratamiento control que registró $78,85 \%$, mientras que al utilizar el $8 \%$ de dulcamara se registró $76,74 \%$ de rendimiento a la canal.

Durante el período de estudio no se registró mortalidad en los pollos de engorde broilers en ninguno de los tratamientos aplicados, incluido el de control, por lo que se deduce que la dosificación al suministrar extracto de dulcamara en el agua de bebida aplicada resultó adecuado.

En cambio en los pollos que estuvieron bajo el efecto del tratamiento control en un $20 \%$, si se registró morbilidad.

\begin{tabular}{|l|c|c|c|c|c|c|c|}
\hline \multicolumn{1}{|c|}{ Rubros } & Unidad & Cant. & C. Unit. & T0 & T1 & T2 & T3 \\
\hline Pollos & pollo & 100 & 0,7 & 17,50 & 17,50 & 17,50 & 17,50 \\
\hline Balanceado & $\mathrm{lb}$ & 1040 & 0,325 & 84,50 & 84,50 & 84,50 & 84,50 \\
\hline Dulcamara & $\mathrm{ml}$ & 1008 & 0,01 & & 1,68 & 3,36 & 5,04 \\
\hline Antibiótico & $\mathrm{g}$ & 250 & 8 & 8,00 & 0,00 & 0,00 & 0,00 \\
\hline Vacunas & dosis & 400 & 0,04 & 4,00 & 4,00 & 4,00 & 4,00 \\
\hline Depreciación & periodo & & & 2,00 & 2,00 & 2,00 & 2,00 \\
\hline Servicios Básicos & U.S.D. & & & 3,00 & 3,00 & 3,00 & 3,00 \\
\hline Mano de obra & U.S.D. & & & 5,00 & 5,00 & 5,00 & 5,00 \\
\hline Total Egresos & 124,00 & 117,68 & 119,36 & 121,04 & & & \\
\hline Pollos vivos & pollo & & & 25 & 25 & 25 & 25 \\
\hline Peso & lb & & & 5,66 & 5,93 & 5,90 & 5,84 \\
\hline Cantidad Carne & lb & & & 141,58 & 148,18 & 147,50 & 146,04 \\
\hline Precio & U.S.D. & & & 1,10 & 1,10 & 1,10 & 1,10 \\
\hline Total Ingresos & & & & 155,74 & 162,99 & 162,25 & 160,65 \\
\hline Beneficio / Costo & U.S.D. & & & 1,26 & 1,39 & 1,36 & 1,33 \\
\hline Utilidad & U.S.D. & & & 31,74 & 45,31 & 42,89 & 39,61 \\
\hline Costo Unitario & U.S.D. & & & 0,88 & 0,79 & 0,81 & 0,83 \\
\hline
\end{tabular}

Tabla 2. Cálculo costo / beneficio.

En cuanto al parámetro económico, la utilización 4, 8 y $12 \%$ de dulcamara en el agua de bebida, registró beneficios de 1,39, 1,36 y 1,33 dólares para los tres tratamientos indicados, lo cual significa que por cada dólar invertido se obtiene un beneficio neto de 0,$39 ; 0,36$ y 0,33 dólares, mientras que los pollos que no recibieron el extracto de dulcamara, presentaron un beneficio/costo de 1,26; demostrándose que por cada dólar invertido se obtiene una ganancia de 0,26 dólares que es una rentabilidad baja (Tabla 2).

Considerando éstos, se demuestra un menor gasto y una mayor rentabilidad al utilizar el extracto de dulcamara en la producción avícola, en comparación con el uso de medicamentos convencionales.

\section{DISCUSIÓN}

En la investigación denominada "Evaluación de tres Niveles de Enzima Allzme-SS (Solid State Fermentation) en Dietas para Pollos Cobb 500 y Ross 308” realizada en la granja avícola Barrionuevo ubicada en el Puyo, provincia de Pastaza, se evalúa la aplicación de la enzima Allzme-SS en pollos de engorde, en donde se registró una ganancia de peso de $3.000 \mathrm{~g}$ y $2.900 \mathrm{~g}(8,9)$, valores superiores a los registrados en el presente 
estudio; de la misma manera manifiesta que la ganancia de peso de pollos durante su crecimiento y engorde fueron de 2.297, 2.645, 2.252, 2.256 y 2.624 g respectivamente (10) valores que se encuentran dentro de los establecidos en la presente investigación.

Por otra parte, en el estudio "Evaluación de la Dulcamara (kalanchoe gastonis bonnieri) en la etapa de crecimiento y engorde en pollos broiler en el barrio Santán del cantón Latacunga", se determinó que con la utilización de KGB al 1,5\% el peso máximo de pollos fue de $3.691,1 \mathrm{~g}$ y el peso mínimo de 2.923,05 g (11), valores superiores a los determinados en este estudio.

Otra investigación importante es "Efecto de la suplementación de dos tipos de Fitasas en pollos, sobre desempeño y metabolismo en zonas de altura" se evaluó dos tipos de fitasas (sólida y liquida) para evaluar el desempeño productivo y metabólico en pollos COBB 508, donde se registran pesos a la canal de $1.950 \mathrm{~g}$ (12), siendo inferior al registrado en el presente estudio.

Cabe mencionar la información obtenida del estudio "Aceites esenciales y fenoles de Allium cepa Var. Red creole (Cebolla Morada) en la producción de pollos broiler" desarrollado en el cantón Guano, provincia de Chimborazo, se evaluó el comportamiento productivo y sanitario de pollos broiler por efecto de tres niveles de extracto de Allium cepa (cebolla morada) en donde en pollos COBB 508 el rendimiento a la canal fue de 70,$70 ; 73,33 ; 74,1 \%$ (13), valores inferiores a los reportados en la presente investigación.

Al utilizar extracto de dulcamara se obtuvieron conversiones de alimento de 1,80 y 2,32 (14), valores extremos a los registrados en el presente estudio, esto quizá se debe a múltiples factores que influyeron en la crianza de pollos como genética y manejo del ambiente externo. La conversión alimenticia en pollos de engorde también se registra 2,17 y 2,10 (15), aunque también se registran conversiones de 1,81 y $1,49(16,17)$. De la misma manera las conversiones alimenticias de los pollos fueron 2,$34 ; 1,69$ y 1,73 (18 - 25), valo- res que se encuentran alrededor de los recopilados en el presente estudio.

Sobre la base de los resultados de esta investigación, se demuestra un menor gasto y una rentabilidad mayor al utilizar el extracto de dulcamara en la producción avícola, en comparación con el uso de medicamentos convencionales.

\section{CONCLUSIONES}

Al utilizar diferentes niveles de extracto de dulcamara en la alimentación de broilers, mejoró el comportamiento productivo en relación con el tratamiento de control, evidenciándose una ganancia de peso de $2.646,88 \mathrm{~g}$, conversión alimenticia 1,97 un peso final de $2.690,88 \mathrm{~g}$, en pollos que recibieron el extracto al $4 \%$.

La aplicación de extracto de dulcamara al 4\%, 8\% y $12 \%$ en el agua de bebida de los pollos no generó mortalidad y morbilidad en pollos broilers, por el contrario se mejoró los parámetros sanitarios y zootécnicos, notándose mejoras significativas.

En cuanto al análisis económico se concluye que los tratamientos en los que se utilizó extracto de dulcamara resultaron ser favorables, obteniendo un beneficio/costo de 1,39 dólares.

Se recomienda elaborar dietas para pollos broilers que incluyan extracto de dulcamara al $4 \%$, por cuanto mejora los comportamientos productivo - sanitarios y económicos de las aves.

Los beneficios de la aplicación de la Kalanchoe gastonis - bonnieri (Dulcamara) en pollos de engorde resultan importantes por lo que debería ser aplicado en otras especies de animales de criadero reemplazando el uso de antibióticos.

\section{AGRADECIMIENTO}

Un agradecimiento a la Escuela Superior Politécnica de Chimborazo por generar el espacio para desarrollar la presente investigación, así como a cada uno de los investigadores autores de este estudio. 
1. Mayla, M., \& Marcelo, L. (2013). Respuesta del cultivo de dulcamara (Kalanchoe gastonis bonnieri) a la aplicación edáfica complementaria con tres tipos de bioestimulantes. Cumbaya, Pichincha. (Tesis de grado.Ingeniero Agrónomo). Universidad Central del Ecuador. Facultad de Ciencias Agrícolas. Carrera de Ingeniería Agronómica. Quito - Ecuador. p 5.

2. Beltrán M.L.M., Pérez A.M.P., Sánchez A.G. \& Ruiz L.H. 2003. Male rat infertility induction/ spermatozoa and epididymal plasma abnormalities after oral administration of Kalanchoe gastonis-bonnieri natural juice. Phytother. Res. 17:315-319.

3. Abdalla S, et al (2017). Efficacy of a Kalanchoe gastonis-bonnieri extract to control bacterial biofilms and dental calculus in dogs.

4. Palumbo A., et al (2017). Potential Therapeutic Effects of Underground Parts of Kalanchoe gastonis-bonnieri on Benign Prostatic Hyperplasia

5. Mayla, M., \& Marcelo, L. (2013). Respuesta del cultivo de dulcamara (Kalanchoe gastonis-bonnierí) a la aplicación edáfica complementaria con tres tipos de bioestimulantes. Cumbayá, Pichincha.

6. Guamushig, F. (2016). Evaluación de la dulcamara (Kalanchoe gastonis bonnieri) en la etapa de crecimiento y engorde en pollos broiler en el barrio Santán del Cantón Latacunga. (Tesis de grado. Ing...). Universidad Técnica de Cotopaxi. Carrera de Medicina Veterinaria. p. 40,70, 97.

7. Freire, M. Berrones, A. 2008. Efecto de diferentes relaciones de Lisina: Energía sobre parámetros zootécnicos en pollos de engorde en Altura. Escuela politécnica del Ejército. Carrera de Ingeniería en Ciencias Agropecuarias. https://repositorio.espe.edu.ec/bitstream/21000/2506/1/T-ESPE-IASA\%20I-003423.pdf.

8. Andrade. V. 2012. Evaluación de tres Niveles de Enzima Allzme-SS (Solid State Fermentation) en Dietas para Pollos Cobb 500 y Ross 308. Tesis de grado. Facultad de Ciencias Pecuarias. ESPOCH. Riobamba, Ecuador.

9. Motoche, M. 2017. Evaluación de balanceados comerciales más la adición de pigmentante natural en la alimentación de pollos broilers en el cantón Morona. Tesis previa a la obtención del título de Ingeniero Zootecnista. ESPOCH - Ext. Morona Santiago.

10. Guatatuca, L. 2016. Comportamiento productivo de los pollos broilers COBB 500 con la aplicación de diferentes niveles de la enzima (allzyme ssf) en el centro de investigación, posgrado y conservación amazónica (CIPCA). Proyecto de investigación. Facultad de Ciencias de la Tierra. UEA - Puyo - Ecuador. Pp 42 - 58.

11. Guamushig, F. (2016). Evaluación de la dulcamara (Kalanchoe gastonis bonnieri) en la etapa de crecimiento y engorde en pollos broiler en el barrio Santán del Cantón Latacunga. (Tesis de grado. Ing...). Universidad Técnica de Cotopaxi. Carrera de Medicina Veterinaria. p. 100, 104, 105.

12. Ortiz, M., y Torres M. 2013. Efecto de la suplementación de dos tipos de fitasas en pollos, sobre desempeño y metabolismo en zonas de altura. Escuela Politécnica del Ejército, Sangolqui. Recuperado el 15 de mayo de 2016 de: http://repositorio.espe.edu.ec/bitstream/21000/7063/1/TESPE-047204.pdf.

13. Sánchez, M. 2016. Aceites esenciales y fenoles de allium cepa var. red creole (cebolla morada) en la producción de pollos broiler. Tesis de Grado. Carrera de Zootecnia, Facultad de Ciencias Pecuarias. Escuela Superior Politècnica de Chimborazo. Riobamba - Ecuador.

14. Chiriboga, A. 2015. Evaluación de tres balanceados energéticos-proteícos comerciales y dos aditivos alimenticios en la alimentación de pollos parrilleros.tumbaco, pichincha. Tesis previa a la obtención del grado de Ingeniero Agronomo. Esceula de Ingenierìa agronómica, Facultad de Ciencias Agrìcolas. Universidad Central del Ecuador. En línea 05/02/2020. (http://www.dspace. uce.edu.ec/bitstream/25000/3240/1/T-UCE-0004-04.pdf).

15. Chiriboga, A. 2015. Evaluación de tres balanceados energéticos-proteícos comerciales y dos aditivos alimenticios en la alimentación de pollos parrilleros.tumbaco, pichincha. Tesis previa a la obtención del grado de Ingeniero Agronomo. Esceula de Ingenierìa agronómica, Facultad de 
Ciencias Agrìcolas. Universidad Central del Ecuador. En línea 05/02/2020. (http://www.dspace. uce.edu.ec/bitstream/25000/3240/1/T-UCE-0004-04.pdf).

16. Bermúdez, H. 2005. Objetivos de la Producción. Santo Domingo de los Achiras, EC. AGRIPAC. s. p. (Guía de producción).

17. Vaca, F.; Araque M. 2006. Evaluación de tres balanceados proteicos energéticos etapa inicial - final, en la alimentación de pollos parrilleros para la venta a las ocho semanas. Sangolquí, Pichincha. Rumipamba.

18. Guevara, I. 2006. Uso de acidificantes intestinales en el control de escherichia coli. Tesis de grado. Facultad de Ing. Zootecnia. ESPOCH - Riobamba - Ecuador. Pp 70 - 75.

19. Pillaga, V. 2010. Evaluación de tres niveles de enzimas (Allizime vegpro) en la alimentación d epollos parrilleros. Tesis de grado. Facultad de Ing. Zootecnia. ESPOCH - Riobamba - Ecuador. Pp $70-75$.

20. Romero, M. 2010. Utilización de promotores Sel-plexl en el engorde de pollos parrilleros. Tesis de grado. Facultad de Ing. Zootecnia. ESPOCH - Riobamba - Ecuador. Pp 56 - 80.

21. Martínez, M., Díaz, M. F., Hernández, Y., Sarmiento, M., \& Sierra, F. 2013. Sustitución de pasta de soya comercial (Glycine max) por harina de frijol de soya germinada y sin germinar en dietas de pollos de engorde. Livestock Research for Rural Development, 25(7).

22. Gonzáles, S., Icochea, E., Reyna, P., Guzmán, J., Cazorla, F., Lúcar, J., ... \& San Martín, V. (2013). Efecto de la suplementación de ácidos orgánicos sobre los parámetros productivos en pollos de engorde. Revista de Investigaciones Veterinarias del Perú, 24(1), 32-37.

23. Casamachin, M. L., Lopez, F. J., \& Ortiz, D. (2007). Evaluación de tres niveles de inclusión de morera (Morus alba) en alimento para pollos de engorde. Biotecnología en el sector agropecuario y agroindustrial, 5(2), 64-71.

24. Shiva, C., Bernal, S., Sauvain, M., Caldas, J., Kalinowski, J., Falcón, N., \& Rojas, R. (2012). Evaluación del aceite esencial de orégano (Origanum vulgare) y extracto deshidratado de jengibre (Zingiber officinale) como potenciales promotores de crecimiento en pollos de engorde. Revista de investigaciones veterinarias del Perú, 23(2), 160-170.

25. Orozco Campo, R., Meleán Romero, R., \& Rodríguez Medina, G. (2004). Costos de producción en la cría de pollos de engorde. Revista Venezolana de Gerencia (RVG), 9(28), 637-659. 